\title{
Dipole Blockade and Quantum Information Processing in Mesoscopic Atomic Ensembles
}

\author{
M. D. Lukin ${ }^{1}$, M. Fleischhauer ${ }^{1,2}$, R.Cote ${ }^{3}$ \\ ${ }^{1}$ ITAMP, Harvard-Smithsonian Center for Astrophysics, Cambridge, MA 02138 \\ ${ }^{2}$ Fachbereich Physik, Universität Kaiserslautern, D-67663 Kaiserslautern, Germany \\ ${ }^{3}$ Physics Department, University of Connecticut, Storrs, Connecticut 06269 \\ L. M. Duan, D. Jaksch, J. I. Cirac and P. Zoller \\ Institut für Theoretische Physik, Universität Innsbruck, A-6020 Innsbruck, Austria
}

(May 28, 2018)

\begin{abstract}
We describe a technique for manipulating quantum information stored in collective states of mesoscopic ensembles. Quantum processing is accomplished by optical excitation into states with strong dipole-dipole interactions. The resulting "dipole blockade" can be used to inhibit transitions into all but singly excited collective states. This can be employed for a controlled generation of collective atomic spin states as well as non-classical photonic states and for scalable quantum logic gates. An example involving a cold Rydberg gas is analyzed.
\end{abstract}

PACS numbers 03.67.-a, 42.50.-p, 73.23.-b 
Recent advances in quantum information science have opened a door for a number of fascinating potential applications ranging from the factorization of large numbers and secure communication to spectroscopic techniques with enhanced sensitivity. But the practical implementation of quantum processing protocols such as quantum computation requires coherent manipulation of a large number of coupled quantum systems which is an extremely difficult task [1]. Challenges ranging from a long-time storage of quantum information to scalable quantum logic gates are by now well known. It is generally believed that precise manipulation of microscopic quantum objects is essential to implement quantum protocols. For example in most of the potentially viable candidates for quantum computers an exceptional degree of control over sub-micron systems is essential for performing single-bit operations and the two-bit coupling is accomplished by interactions between nearest neighbors [2]. Related techniques are also being explored that involve photons to connect qubits [3], and to construct potentially scalable quantum networks [4]. However, since the single-atom absorption cross-section is very small, reliable coupling to light requires high-finesse micro-cavities [5].

In the present Letter we describe a technique for the coherent manipulation of quantum information stored in collective excitations of mesoscopic many-atom ensembles. This is accomplished by optically exciting the ensemble into states with a strong atom-atom interaction. Specifically, we consider the case involving dipole-dipole interactions in an ensemble of cold atoms excited into Rydberg states. Under certain conditions the level shifts associated with these interactions can be used to block the transitions into states with more than a single excitation. The resulting "dipole blockade" phenomenon closely resembles similar mesoscopic effects in nanoscale solid-state devices [6]. In the present context it can take place in an ensemble with a size that can exceed many optical wavelengths. Combined with the exceptional degree of control that is typical for quantum optical systems and long coherence times, this allows to considerably alleviate many stringent requirements for the experimental implementation of various quantum processing protocols. In particular, we show that this technique can be used to i) generate superpositions of collective spin states (or Dicke 
states) in an ensemble; ii) coherently convert these states into corresponding states of photon wavepackets of prescribed direction, duration and pulse-shapes and vice versa using the collectively enhanced coupling to light [9]; and iii) perform quantum gate operations between distant qubits. Corresponding applications including (i) sub-shot noise spectroscopy and atom interferometry [7], (ii) secure cryptography protocols [8], and (iii) scalable quantum logic devices can be foreseen. In general, no strongly coupling micro-cavities and no single particle control is required to implement computation and communication protocols. We further anticipate that the approach can be applied to a variety of interacting many-body systems ranging from trapped ions to specifically designed semiconductor structures.

The basic element of the present scheme is an ensemble of $N$ identical multi-state atoms (Fig.1) contained in a volume $V$. Using well-developed techniques all atoms can be trapped and prepared in a specific sub-level $\left(\left|g_{i}\right\rangle, i=1 \ldots N\right)$ of the ground state manifold. Relevant states of each atom include: a pair of meta-stable sub-levels of the ground state manifold $\left|q_{i}\right\rangle$ that are used for long-time storage of qubits (storage states) and long-lived Rydberg states $\left|r_{i}\right\rangle,\left|p_{i}^{\prime}\right\rangle,\left|p_{i}^{\prime \prime}\right\rangle$. Additional Rydberg sublevels as well as lower electronic excited states can be used for specific applications. We assume modest atomic densities, such that interactions between atoms can safely be neglected whenever they are in the sub-levels of the ground state. This also implies long coherence lifetimes - up to few seconds - associated with the storage sub-levels. However when excited into the Rydberg states the atoms interact strongly with each other due to the presence of resonant long-wavelength dipole-dipole interactions.

The manipulation of atoms is done by light fields of different frequencies and polarizations, which illuminate the entire ensemble and excite all atoms with equal probability. Throughout the paper we will assume that hyperfine sublevels of the ground state and Rydberg states are excited in a Doppler free configuration, which can be done using two-photon processes. The Hamiltonian describing these interactions can be written in terms of collective operators $\hat{\Sigma}_{\mu \nu}=\sum_{i}\left|\mu_{i}\right\rangle\left\langle\nu_{i}\right| / \sqrt{N}$, where $\left|\mu_{i}\right\rangle,\left|\nu_{i}\right\rangle$ denote single-atom eigenstates. As a consequence only symmetric collective states are involved in the process. The relevant ones are here the ground state $|\mathbf{g}\rangle \equiv\left|\mathbf{g}^{N}\right\rangle=\left|g_{1}\right\rangle \ldots\left|g_{N}\right\rangle$, the $n$-times excited 
storage states $\left|\mathbf{q}^{n}\right\rangle \equiv\left|\mathbf{g}^{N-n}, \mathbf{q}^{n}\right\rangle=\sqrt{(N-n) ! N^{n} /(N ! n !)}\left(\hat{\Sigma}_{g q}^{+}\right)^{n}|\mathbf{g}\rangle$, and the Rydberg states $\left|\mathbf{r}^{n}\right\rangle \equiv\left|\mathbf{g}^{N-n}, \mathbf{r}^{n}\right\rangle=\sqrt{(N-n) ! N^{n} /(N ! n !)}\left(\hat{\Sigma}_{g r}^{+}\right)^{n}|\mathbf{g}\rangle$. For a sufficiently small number $n \ll N$, these are bosonic excitations, since $\left[\hat{\Sigma}_{\mu \nu}, \hat{\Sigma}_{\mu \nu}^{+}\right] \approx 1$.

We now discuss the interaction between collective atomic excitations. We assume, for a moment, that the atoms are "frozen" in space. To be specific we here consider excitation "hopping" via resonant dipole-dipole interactions between Rydberg atoms (Föster process [11]) as illustrated in Fig. 1b [12]. The energy separation between the optically excited Rydberg state $\left|r_{i}\right\rangle$ and the pair of the sub-levels of different parity $\left|p_{i}^{\prime}\right\rangle,\left|p_{i}^{\prime \prime}\right\rangle$ is adjusted (using e.g. electric fields) such that $E_{r}-E_{p^{\prime}}=E_{p^{\prime \prime}}-E_{r}$. In this case, any pair of atoms excited in the $\left|r_{i}\right\rangle\left|r_{j}\right\rangle$ states would undergo a hopping transition into the states $\left|p_{i}^{\prime}\right\rangle\left|p_{j}^{\prime \prime}\right\rangle$ and $\left|p_{i}^{\prime \prime}\right\rangle\left|p_{j}^{\prime}\right\rangle$ etc., resulting in a splitting of the excited Rydberg components. For a system with three effective Rydberg states (Fig.1b) the relevant process is described by the Hamiltonian:

$$
\hat{V}_{d}=\hbar \sum_{i>j} \kappa_{i j}\left|r_{i}\right\rangle\left|r_{j}\right\rangle\left(\left\langle p_{i}^{\prime}\right|\left\langle p_{j}^{\prime \prime}\right|+\left\langle p_{j}^{\prime}\right|\left\langle p_{i}^{\prime \prime}\right|\right)+\text { h.c. },
$$

where $\hbar \kappa_{i j} \sim \wp_{r p^{\prime}} \wp_{r p^{\prime \prime}} / r_{i j}^{3}, \wp_{k l}$ being the dipole matrix elements for the corresponding transitions and $r_{i j}$ is the distance between the two atoms [13]. In general this interaction does not affect the singly-excited collective states (e.g. $\hat{V}_{d}|\mathbf{r}\rangle=0$ ) but leads to a splitting of the levels when two or more atoms are excited. In particular, the collective eigenstate with atoms $i$ and $j$ being excited and all other atoms in the ground or storage states

$$
\begin{aligned}
\left| \pm_{\mathbf{i j}}^{\mathbf{2}}\right\rangle & =\frac{1}{\sqrt{2}}\left[\left|g_{1} \ldots r_{i} \ldots r_{j} \ldots g_{N}\right\rangle\right. \\
& \left. \pm\left(\left|g_{1} \ldots p_{i}^{\prime} \ldots p_{j}^{\prime \prime} \ldots g_{N}\right\rangle+\left|g_{1} \ldots p_{j}^{\prime} \ldots p_{i}^{\prime \prime} \ldots g_{N}\right\rangle\right) / \sqrt{2}\right]
\end{aligned}
$$

are split by $\hbar \kappa_{i j}$. It follows that for an ensemble contained in a finite volume $V$ the manifold of doubly excited states has a minimum splitting of order $\bar{\kappa}=\wp_{r p^{\prime}} \wp_{r p^{\prime \prime}} /(\hbar V)$ (Fig.2). Thus if $\bar{\kappa}$ is much larger than the linewidth $\gamma_{r}$ of the Rydberg state, resonant excitations from the singly to the doubly-excited state are strongly suppressed. This is the essence of the dipole blockade. 
Turning to the interaction of such many-atom systems with light we first discuss how to create single collective qubits. When the splittings of the doubly-excited Rydberg states are sufficiently large, a weak light field tuned to the single-atom resonance frequency can excite the transition between the ground state and the first collective state $|\mathbf{r}\rangle$. But due to the splitting of the states $\left| \pm_{\mathbf{i j}}{ }^{2}\right\rangle$ successive transitions into these and higher states are strongly inhibited. Hence if the atomic system is initially in its ground state $|\mathbf{g}\rangle$ the evolution is given by the effective two-level dynamics

$$
\left[\begin{array}{c}
|\mathbf{g}(t)\rangle \\
|\mathbf{r}(t)\rangle
\end{array}\right]=\left[\begin{array}{cc}
\cos \theta(t) & -i \sin \theta(t) \\
i \sin \theta(t) & \cos \theta(t)
\end{array}\right]\left[\begin{array}{c}
|\mathbf{g}(0)\rangle \\
|\mathbf{r}(0)\rangle
\end{array}\right]
$$

where $\theta(t)=\sqrt{N} \int_{0}^{t} \Omega(\tau) / 2 d \tau$. The ensemble displays Rabi-oscillations only between ground and singly excited Rydberg state with a collective Rabi rate $\sqrt{N} \Omega$. There are obviously several conditions that restrict the validity of Eq.(3). First of all, the Hamiltonian evolution takes place for times $t$ much shorter than the dephasing time $\gamma_{\mathbf{r}}{ }^{-1}$. Secondly, there is a finite probability to populate the doubly excited states $\left| \pm_{\mathbf{i j}}, 2\right\rangle$ that scales as $\sim \bar{\kappa}^{-2}$. For example, at a time $t=T$ where a single collective excitation is generated (i.e. where $\theta(T)=\pi$ ) the probabilities of errors due to populating the doubly excited states and dephasing scale, respectively, as

$$
p_{\text {doub }} \sim \frac{1}{N^{2}} \sum_{i . j} \frac{1}{\kappa_{i j}^{2} T^{2}} \approx \frac{1}{4 \pi(\bar{\kappa} T)^{2}}, \quad p_{\text {deph }} \sim \gamma_{\mathbf{r}} T .
$$

Hence, the system can be driven into superpositions of collective states $\alpha_{0}|\mathbf{g}\rangle+\alpha_{1}|\mathbf{r}\rangle$. The single-quantum excitation can now be stimulated into a storage sub-level (e.g. $|\mathbf{q}\rangle$ ) by a $\pi$-pulse $\left(\int \Omega_{q}(\tau) d \tau=\pi\right)$ and we can associate a qubit with the state $\alpha_{0}|\mathbf{g}\rangle+\alpha_{1}|\mathbf{q}\rangle$. As indicated in Fig. 2, this procedure can be generalized to the generation of higher order collective states or their superpositions using a sequence of properly timed pulses. For example, the sequence of two pulses $\sqrt{N-n} \int \Omega(\tau) d \tau=\pi$ and $\sqrt{n+1} \int \Omega_{q}(\tau) d \tau=\pi$ results in $\left|\mathbf{q}^{n}\right\rangle \rightarrow\left|\mathbf{q}^{n+1}\right\rangle$. Note that under the conditions of dipole blockade the transitions into collective Rydberg states other than $\left|\mathbf{r}, \mathbf{q}^{m}\right\rangle$ are always inhibited. The scaling of the error probability in each step is given by Eq.(4). To prove that a synthesis of arbitrary 
superpositions $\left|\Psi_{n}\right\rangle=\sum_{m=0}^{n} \alpha_{m}\left|\mathbf{q}^{m}\right\rangle$ is possible we note that the inverse procedure, i.e. the evolution from a known state $\left|\boldsymbol{\Psi}_{n}\right\rangle$ into the ground state $|\mathbf{g}\rangle$ can easily be constructed. In particular, $2 n$ pulses of proper duration can be used to sequentially empty the states $\left|\mathbf{q}^{n}\right\rangle,\left|\mathbf{r} ; \mathbf{q}^{n-1}\right\rangle,\left|\mathbf{q}^{n-1}\right\rangle$ etc, and the system would evolve into its ground state. Since this evolution is unitary a reverse sequence results in $|\mathrm{g}\rangle \rightarrow\left|\Psi_{n}\right\rangle$.

Before proceeding we note several important properties of collective spin states generated by means of the dipole blockade. First of all, these states are precisely of the from required to maximize sensitivity of atomic clocks [7] beyond the quantum limit. Secondly, by using standard techniques of atom optics it is possible to couple the atoms in a specific internal state out of the trap. When these techniques are applied to the atoms in state $|q\rangle$, the states with a desired number of atoms can be coupled out. In addition to a number of important potential applications for entanglement engineering in atom interferometers, such an "atom gun" can make fascinating experiments involving spatially delocalized Schrödinger cat states possible. Finally, the spin states $\left|\mathbf{q}^{n}\right\rangle$ exhibit a collectively enhanced coupling to light, when excited in a proper two-photon configuration. Consequently, the state $\left|\Psi_{n}\right\rangle$ can be transfered from the spin degrees of freedom to the optical field [9]. If the optical thickness of the cloud at the resonance wavelength is sufficiently high, this allows to generate wavepackets on demand with desired properties such as quantum states, pulse shapes and duration without the use of high-Q cavities as necessary in single-atom cavity-QED [14]. The quantum state of these wavepackets can be transferred to distant ensembles [9] thereby making quantum networking possible.

The dipole blockade mechanism also facilitates quantum logic operations. For instance, in proof-of-principle experiments qubits stored in few- $\mu m$ spaced atomic clouds can be entangled if the transitions in one ensemble are inhibited when a collective Rydberg state is excited in a second ensemble. In essence, this scheme utilizes the same principle as that of the Ref. |15] with the single-particle excitations replaced by collective qubits stored e.g. in $\{|\mathbf{g}\rangle,|\mathbf{q}\rangle\}$. In what follows we discuss another approach that allows for such operations between distant ensembles, which is instrumental for scalability. In this approach the qubit states of two dif- 
ferent ensembles $\left\{|\mathbf{g}\rangle_{1},|\mathbf{q}\rangle_{1}\right\}$ and $\left\{|\mathbf{g}\rangle_{2},|\mathbf{q}\rangle_{2}\right\}$ are first transferred to two hyperfine sublevels of one ensemble (as shown in Fig.3) via mapping to light fields: $\left.\left|\mathbf{q}_{1} \rightarrow\right| \mathbf{q}_{+}\right\rangle,|\mathbf{q}\rangle_{2} \rightarrow\left|\mathbf{q}_{-}\right\rangle$. A universal logic gate is then performed in three steps. First, all atoms in the storage states $\left|q_{-}\right\rangle$are excited into a processing Rydberg state $\left|r_{-}\right\rangle$by a $\pi$-pulse, $\int_{0}^{T} \Omega_{-}(\tau) d \tau=\pi$. Second, the atoms in the storage states $\left|q_{+}\right\rangle$are excited into a processing state $\left|r_{+i}\right\rangle$ and back by a $2 \pi$-pulse, $\int_{0}^{T} \Omega_{+}(\tau) d \tau=2 \pi$. This results in a phase shift of $\pi$ for the collective state $\left|\mathbf{q}_{+}\right\rangle$. Note, however, that transitions into the doubly excited states $\left|g_{1} \ldots r_{+i} \ldots r_{-j} \ldots g_{N}\right\rangle_{1}$ are inhibited due to the dipole blockade. As a result, the atoms in the collective state $\left|\mathbf{r}_{-}, \mathbf{q}_{+}\right\rangle$ do not acquire any phase rotation. In a final step, the atoms in state $\left|r_{-}\right\rangle$are stimulated back into the storage sublevels resulting in a total phase shift of $\pi$ for the states $\left|\mathbf{q}_{ \pm}\right\rangle$and $\left|\mathbf{q}_{+}, \mathbf{q}_{-}\right\rangle$. Hence, a conditional phase shift

$$
|\mathbf{g}\rangle \rightarrow|\mathbf{g}\rangle,\left|\mathbf{q}_{ \pm}\right\rangle \rightarrow-\left|\mathbf{q}_{ \pm}\right\rangle,\left|\mathbf{q}_{+}, \mathbf{q}_{-}\right\rangle \rightarrow-\left|\mathbf{q}_{+}, \mathbf{q}_{-}\right\rangle
$$

is obtained. The gate is completed by sending the qubits back to the original registers. The leading sources of errors are again due to the finite probability of populating the doubly excited states $p_{d o u b}$, and due to dephasing of Rydberg transitions $p_{d e p h}$, given by Eq.(四).

We next discuss a number of important practical issues associated with the present technique. First of all the decoherence of collective excitations is of particular importance here: the qubit states such as $\left|\mathbf{q}_{ \pm}\right\rangle,|\mathbf{r}\rangle$ are, in fact, entangled superpositions of $N$ singleparticle states and these might be expected to be extremely fragile. This is not the case, since the symmetric entangled states involved are known to be very robust against e.g. particle loss [16]. As a result, collective dephasing rates are equal to the corresponding single-particle rates, if the dephasing is dominated by single-particle rather than collective processes. The last assumption is well justified as long as the average inter-atomic distance is below the reduced optical wavelength $\lambda /(2 \pi): N / V \leq(2 \pi / \lambda)^{3}$ [17]. Errors due to atom-number fluctuations $(\Delta N)$ are negligible, provided that $\Delta N \ll N$. Finally, because the states that are involved in strong atomic interactions are, in the ideal limit, never populated, the present approach avoids mechanical interaction between atoms and leaves the qubit degrees 
of freedom decoupled from atomic motion [15].

Present day magnetic or optical traps allow one to contain about $N \sim 10^{4}$ cold alkali atoms ( $\mathrm{Li}$, Cs or $\mathrm{Rb}$ ) within $V<(10 \mu \mathrm{m})^{3}$. For the atoms excited to Rydberg states ( $n \sim 50$ ), typical coherence lifetimes limited by radiative relaxation and black-body radiation correspond to $\gamma_{\mathbf{r}}<10 \mathrm{kHz}$, whereas dipole-dipole interactions correspond to values of $\bar{\kappa}$ in the range of 10-100 MHz. This implies that for excitation on a fast time scale such as $T<100$ ns, the probability of loss $p_{\text {doub }}, p_{\text {loss }}<1 \%$. Such ensembles are opaque near the resonant optical transitions of the $D_{1,2}$ absorption lines, thereby making efficient coupling to light possible. In a potentially scalable approach one could employ arrays of such traps created on nanofabricated surfaces [18], in which case the traps could be also coupled using low-Q cavities and low-loss fiber waveguides. Finally, since the atomic motion is never coupled to the qubits, the temperature is only limited by the requirement that the atomic distribution should not change significantly on the time scale of the gate operation $T$ ("frozen" gas approximation). This is the case even for temperatures as high as a few $m K$.

In summary, we have shown that the dipole blockade can be used for coherent manipulation and entanglement of collective excitations in mesoscopic ensembles of cold atoms. We anticipate a number of potential applications ranging from atomic clocks and secure quantum communication to quantum computation with mesoscopic atomic samples.

We thank E. Eyler, P. Gould, T. Gallagher and V. Kharchenko for many stimulating discussions. This work was supported by the Austrian Science Foundation, the Europe Union project EQUIP, the ESF, the European TMR network Quantum Information, the NSF through the grant to the ITAMP and the NSF ITR program. M.F. thanks the Deutsche Forschungsgemeinschaft for financial support and ITAMP for the hospitality during his stay. 


\section{REFERENCES}

[1] See e.g.: D. P. DiVincenzo, Science 270, 255 (1995); C. H. Bennett, Phys. Today 48, No. 10, 24 (1995); A. Ekert and R. Josza, Rev. Mod. Phys. 68, 733 (1996).

[2] See J. Gruska, Quantum Computing (McGraw-Hill, London, 1999); A. Steane, Rep. Prog. Phys. 61, 117 (1998).

[3] T. Pelizzari, S. A. Gardiner, J. I. Cirac, and P. Zoller, Phys.Rev.Lett. 75, 3788 (1995).

[4] J. I. Cirac, P. Zoller, H. Mabuchi and H. J. Kimble, Phys.Rev.Lett. 78, 3221 (1997).

[5] Q.A.Turchette et al., Phys.Rev.Lett. 75, 4710 (1995).

[6] See e.g. Mesoscopic phenomena in Solids, B.L.Altshuler, P.A.Lee, and R.Webb, Eds., (Elsevier,Amsterdam, 1991).

[7] D.J. Wineland, et al., Phys.Rev.A 46, R6797 (1992); J. Bollinger et al., ibid 54, 4649 (1996). S. F. Huelga, et al. Phys. Rev. Lett. 79, 3865 (1997); P. Boyer and M. A. Kasevich, Phys. Rev. A 56, R1083 (1997).

[8] Deterministic single-photon sources are crucial for physical implementation of a secure BB84 quantum cryptography protocol; see. e.g. N. Luetkenhaus, Phys. Rev. A, 61, $052304(2000)$.

[9] M. D. Lukin, S. F. Yelin, and M. Fleischhauer, Phys. Rev. Lett., 84, 4232 (2000); M. Fleischhauer and M. D. Lukin, Phys. Rev. Lett. 84, 5094 (2000); L.M. Duan, J.I. Cirac, and P. Zoller, unpublished.

[10] T.F. Gallagher, Rydberg atoms, (Cambridge University Press New York 1994).

[11] Th. Föster, in Modern Quantum Chemistry, O. Sinanoglu Ed., (Academic Press, NewYork, 1996).

[12] Such processes have been observed in a cold Rydberg matter, see e.g. I. Mourachko, et al., Phys. Rev. Lett. 80253 (1998); W.R. Anderson et al., ibid., 249. 
[13] E.g. in a case when the Rydberg $S$-state is excited by light the pair-coupling strength $\kappa_{i j}$ depends only on the separation of the atoms for symmetry reasons.

[14] F. De Martini et al., Phys. Rev. Lett. 76, 900 (1996); C. K. Law and H. J. Kimble, J. Mod. Opt.44, 2067 (1997); M. Hennrich et al., Phys. Rev. Lett, in press.

[15] These issues have been discussed in detail for quantum gates based on individual Rydberg atoms, D. Jaksch et al, Phys. Rev. Lett. 852208 (2000).

[16] W. Dür, G. Vidal and J. I. Cirac, quant-ph/0005115.

[17] Levels can also be shifted due to interaction of the excited Rydberg atoms with ground state atoms. The magnitude of these shifts is at most few hundred $\mathrm{KHz}$ at densities $N / V \sim 10^{13} \mathrm{~cm}^{-3}$. These shifts can be compensated by a small detuning since the broadening is expected to be much smaller than the shift at low temperatures.

[18] J. Reichel, W. Hänsel, and T. W. Hänsch , Phys. Rev. Lett. 833398 (1999); R.Folman et al., Phys. Rev. Lett. 844749 (2000). 


\section{FIGURES}

(a)

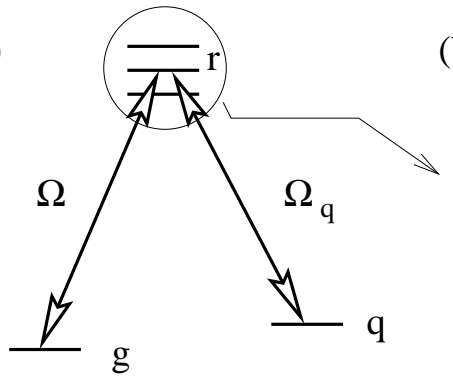

(b)

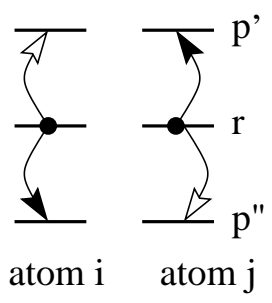

FIG. 1. (a) Relevant single-atom levels for quantum information storage $(q)$ and processing $\left(r, p^{\prime}, p^{\prime \prime}\right)$ can be accessed by optical fields. $\Omega, \Omega_{q}$ are Rabi-frequencies. (b) Resonant atom-atom interaction causes excitation hopping between atom pairs and results in collective level splitting. Arrows indicate atomic transitions in the hopping process.
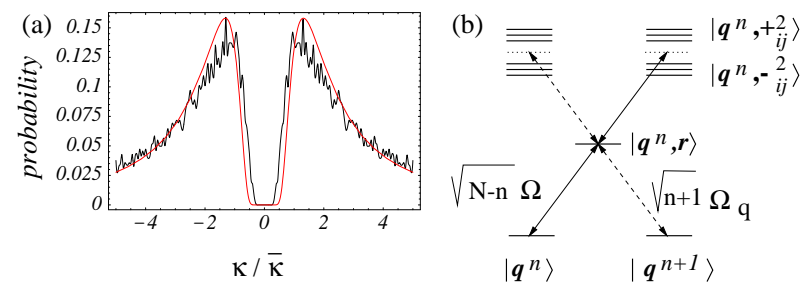

FIG. 2. (a) Probability distribution for frequency splittings of manifold of doubly-excited Rydberg states. Shown is a Monte-Carlo simulation for $3 \times 10^{4}$ positions in a rectangular box and analytical approximation for a random gas $p(x)=\sqrt{2} \pi \exp \left[-\pi^{3} / 18 x^{2}\right] /\left(6 x^{2}\right), x=\kappa / \bar{\kappa}$. (b) Successive generation of Fock states $\left|\mathbf{q}^{n}\right\rangle$ using dipole-blockade.
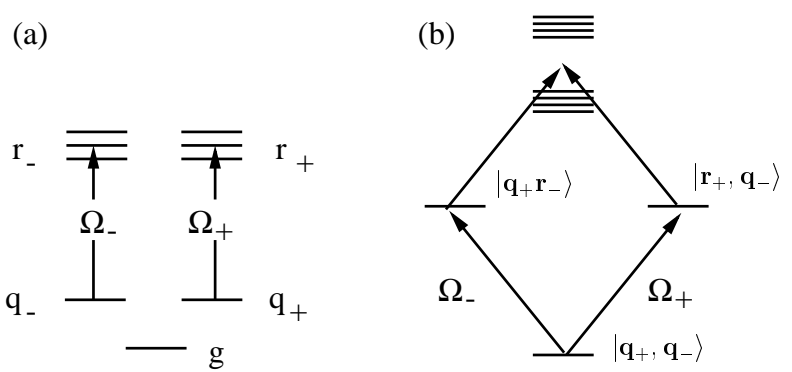

FIG. 3. (a) Level scheme for generation of conditional phase shifts. (b) Coupling scheme within the manifold of doubly excited collective states. 